\title{
Low-speed electric drive of the solar battery pivot device
}

\author{
Dmitry Yakimovsky, Dmitrii Polozhentcev and Daniel Dzhukich* \\ JSC “Command devices research institute”, St. Petersburg, Russia
}

\begin{abstract}
The problem of designing a control system for monitoring angular velocities and accelerations has been considered with regard to a low-speed actuating electric drive application. The block diagram for the electric drive, which weight-dimension characteristics are subject to strict requirements, is presented herein. The results of experimental development of the actuating electric drive are discussed below.
\end{abstract}

\section{Introduction}

Most spacecraft have appendages, like solar arrays or antennas, to be deployed, and in some cases these appendages need to be continuously rotated or repointed during the mission [1]. Solar arrays are rotated to trace the source of solar radiation corresponding to the spacecraft's rotation in order to generate power at optimal levels [2]. Actuators are widely used for this purpose, and although currently there exist various types of state-of-the-art actuators based on smart material technology (piezoelectric actuators, shape memory alloys, etc.), conventional technologies, namely electric motors (stepper motors, permanent magnet synchronous motors, etc.) in combination with gearboxes, remain the most preferred ones with a lot of research dedicated to them [1, 3-6].

Modern actuating electric drive (AED) of the solar battery pivot device (SBPD) of spacecraft is designed for slow operational rotation velocity of the output shaft (OS), which may be as low as tenths, hundredths or even thousandths of a degree per second. Other features of the SBPD AED include restrictions for OS rotation acceleration and the high dry friction torque occurring in the contact brush device transmitting power from the solar battery to the spacecraft power supply system. Tough restrictions on weight and dimensions of onboard drives, the requirement to withstand the effects of outer space and ensure long term service of spacecraft significantly affect the design and structure of the electric drive. Extensive research has been conducted to achieve optimal operational results, overcome various restrictions and solve arising issues. For example, research [7] concludes that a system with fuzzy PID control eliminates "flat top" position tracking and "dead zone" speed tracking, which are generated by traditional PID, and thereby decreases the effect of friction on the performance of the servo system. Other researches advocate the need for combined control systems. Paper [8] demonstrates that the use of a combined position and speed controller results in better output speed accuracy, lower current consumption and lower temperature.

The purpose of this paper is to create a SBPD AED based on the brushless motor with a single lowresolution (16-18 bits) induction angle sensor. A drive creating angular rotation velocity of the OS within the range of $\pm(0 ; 0.0039 \ldots 0.5) \%$, with discreteness of NMT $0.000244 \%$, is considered as an example. The OS rotation acceleration value must fall within the range of 0.0012 to $0.01 \% / \mathrm{s}^{2}$, and inaccuracy of the resulting steady state angular velocity may not exceed $10 \%$ for the whole range. Shaft dry friction torque may reach $0.5 \mathrm{~N} \cdot \mathrm{m}$, while external disturbing torque varies within the range from -0.3 to $0.3 \mathrm{~N} \cdot \mathrm{m}$. To realize speed control, angle loop PD regulator was applied which was supplemented with two components: gradually increasing speed for acceleration restriction and an additional non-linear link ensuring almost constant overcoming of dry friction. This approach proved suitable for achieving the given parameters of the system.

\section{Design and modeling}

\subsection{Rotation velocity control}

The velocity control system relies on availability of a velocity indicator. Rotation velocity can be calculated from the angle sensor readings. The risk of error in steady state can be minimized by using a PI-controller within the control loop.

However, if an encoder angle sensor is used in the feedback loop, a different approach to angular rotation velocity control appears to be more practical: namely integration of velocity assigning signal followed by operation in tracker mode with control by angular position of the OS. In this case, PD-controller may be used for angle control $[9,10]$.

Block diagram of such SBPD AED operating in tracker mode based on the linearly varying angle is shown in Figure 1.

\footnotetext{
* Corresponding author: info@niikp.spb.ru
} 


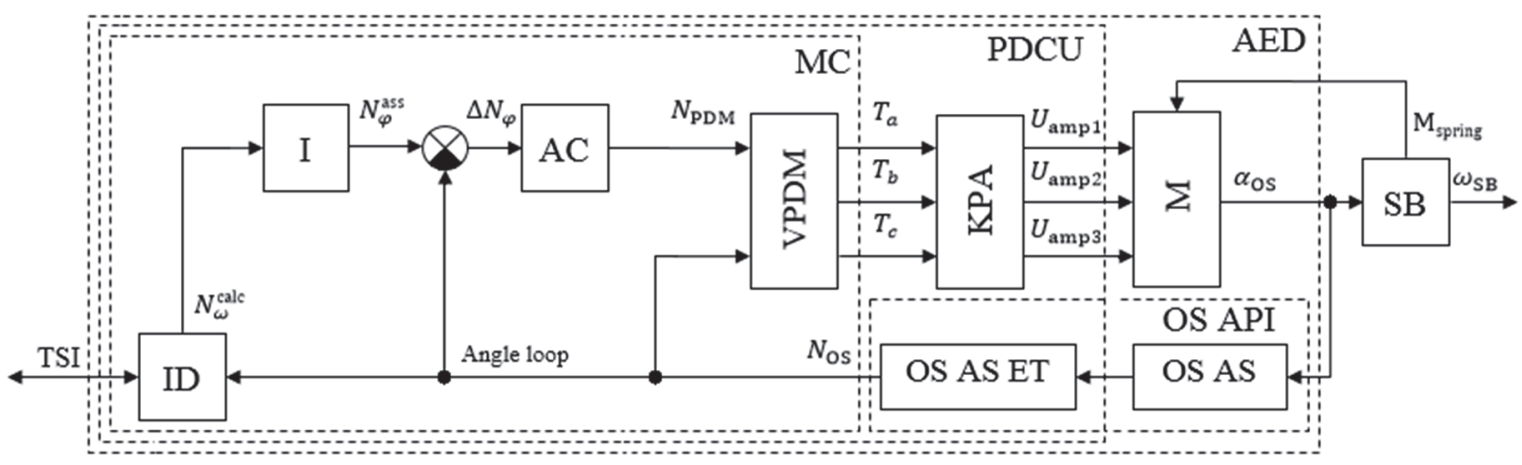

Fig. 1. Block diagram of AED operating in tracker mode based on angle readings.

Where I is velocity integrator; $\mathrm{AC}$ is angle controller; VPDM is vector pulse-duration modulator; KPA is key power amplifier; $\mathrm{M}$ is motor; SB is solar battery; OS AS is OS angle sensor; OS AS ET is electronic transducer of the output shaft angle sensor; ID is interface device; MC is microcontroller; PDCU is pivot device control unit; AED is actuating electric drive; $N_{\omega}$ is code of the assigned angular rotation velocity of the OS; $N_{\varphi}^{\text {assigned (ass) }}$ is calculated code of OS rotation; $\Delta N_{\varphi}$ is angle loop error signal; $N_{\text {pulse-duration modulator (PDM) }}$ is VPDM code input value; $T_{a} ; T_{b} ; T_{c}$ is duration of VPDM signals controlling KPA; $U_{\text {amplitude }(a m p) 1} ; U_{a m p 2}$; $U_{a m p 3}$ is amplitude values of motor feed linear voltages; $\alpha_{\text {output shaft (OS) }}$ is

OS angular position data; $\omega_{\text {solar battery }(S B)}$ is SB angular velocity values; $M_{\text {spring }}$ is spring torque created by SB; $N_{O S}$ is code of measured rotation angle of OS; TSI is trunk serial interface.

Velocity integration coefficient is calculated by the following formula:

$$
\mathrm{C}_{\omega}=\frac{\mathrm{C}_{\text {slope }(\mathrm{sl})}}{\delta},
$$

where $\mathrm{C}_{\mathrm{sl}}$ is "code-velocity" characteristics slope, $\delta$ is "angle-code" conversion discreteness of angle sensor.

"Code-velocity" characteristics slope is defined as:

$$
\mathrm{C}_{\omega}=\frac{\omega_{\text {ass.max }}}{2^{n}-1},
$$

where $\omega_{\text {ass.max }}$ is maximum assigned velocity, $n$ is number of used bits of register that stores the assigned velocity value (not considering the sign bit).

The outputs of mathematical model of AED operation at the assigned velocity of $0.1 \%$ are shown in Figure 2. It can be seen that the required accuracy of the SBPD OS angular rotation velocity control is attained. However, the acceleration diagram shows multifold exceeding of the OS rotation acceleration in relation to the assigned value.

\subsection{Angular acceleration control}

The easiest way of solving this problem could have been to limit the current amplitude in the actuating motor winding, and thus curtail the resulting torque (i.e. acceleration). However, as friction torque is a variable (i.e. its exact value is not known) that changes with angular position of the OS, it would be impossible to fine-tune such a current regulator.

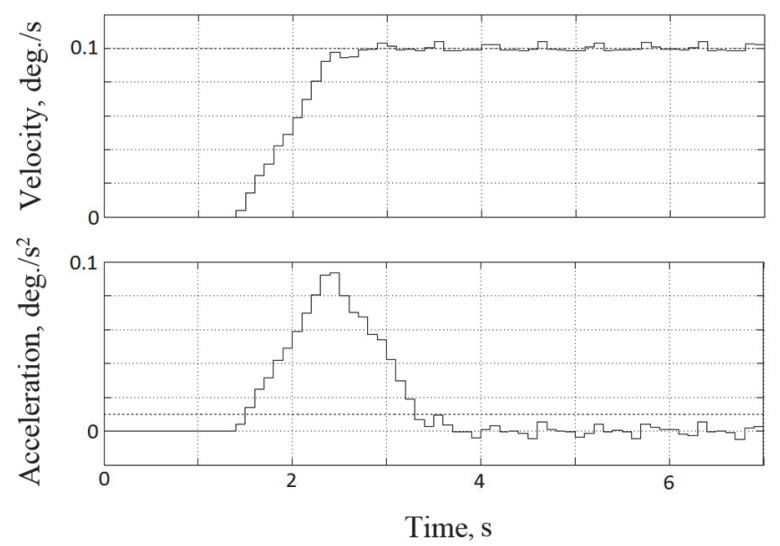

Fig. 2. Outputs of model of AED operation at the assigned velocity of $0.1 \%$.

To solve the problem, a velocity code generator $\left(\mathrm{CG}_{\omega}\right)$ has been integrated into the AED structure, as shown in Figure 3. On receipt of $N_{\omega}$, the velocity code generator generates a linearly increasing (decreasing)

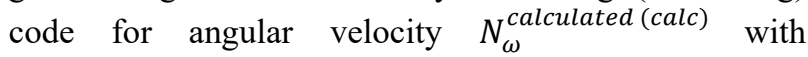
limitation at the level corresponding to the code $N_{\omega}$. In order to keep the OS angular rotation acceleration $\left(\varepsilon_{O S}\right)$ within the range of 0.0012 to $0.01 \% / \mathrm{s}^{2}$, the rate of increasing (decreasing) of calculated code of the motor shaft angular velocity is defined by the following difference equation:

$$
\begin{gathered}
N_{\omega}^{\text {calc }}(n)=k_{\text {generator integration }(G I)} \\
+N_{\omega}^{\text {calc }}(n-1),
\end{gathered}
$$

where $n$ is the reference number of the AED operation cycle; $T_{S}$ is sampling period; $k_{G I}$ is integration factor of generator:

$$
k_{G I}=\varepsilon_{O S} \cdot \frac{1}{\omega_{O S}},
$$

where $\omega_{O S}=N_{\omega}(n) \cdot D_{\mathrm{OS}}$. 


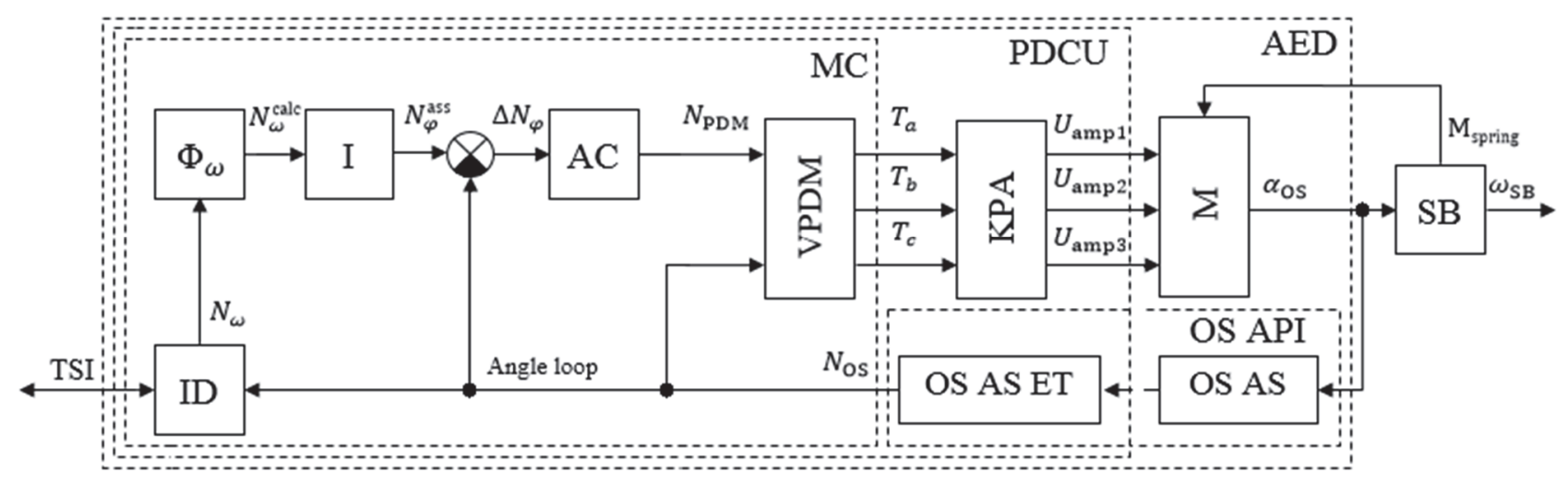

Fig. 3. Block diagram of AED with velocity code generator operating in tracker mode controlled by angle position variations.

In order to keep OS acceleration within the specified range from 0.0012 to $0.01 \% \mathrm{~s}^{2}$, mean $\mathrm{OS}$ angular acceleration was selected on the basis of the modelling results:

$$
\left\{\begin{array}{c}
\varepsilon_{O S}=0.005, \text { at } N_{\omega}>N_{\omega}^{\text {calc }} \\
\varepsilon_{O S}=-0.005 \text { at } N_{\omega}<N_{\omega}^{\text {calc }} \\
\varepsilon_{O S}=0, \text { at } N_{\omega}=N_{\omega}^{\text {calc }}
\end{array}\right.
$$

Then

$$
\left\{\begin{array}{c}
k_{G I}=\frac{\varepsilon_{O S}}{N_{\omega}(n) \cdot D_{O S}} \text {, at } N_{\omega}>N_{\omega}^{\text {calc }} \\
k_{G I}=\frac{-\varepsilon_{O S}}{N_{\omega}(n) \cdot D_{O S}}, \text { at } N_{\omega}<N_{\omega}^{\text {calc }} \\
k_{G I}=0, \text { at } N_{\omega}=N_{\omega}^{\text {calc }}
\end{array}\right.
$$

Outputs of mathematical model of AED operation at the assigned velocity of $0.1 \%$ set by the generator are shown in Figure 4. It can be seen that the required accuracy of control is attained for both angular velocity and angular acceleration of SBPD OS. The time lag between issuing the angular velocity command and start of movement is more than 5 seconds, which is due to the high dry friction torque.

\subsection{Overcoming dry friction torque}

In the considered SBPD, dry friction torque value may be up to $0.5 \mathrm{~N} \cdot \mathrm{m}$ (the value may vary between instruments and depends on OS angular position), which makes $\approx 30 \%$ of rated torque of actuating motor. Such friction torque value may cause a significant delay of OS response to the assigned velocity code. To solve the problem, a non-linear link (NL) within the control loop generates a code $N_{\text {non-linear link (NL) }}$ to increase the rated drive slope within the range of small angular misalignments by adjusting the calculated code $N_{\text {PDM }}$ based on the code sign of calculated angular velocity. Thus, block diagram of the considered AED is modified as shown in Figure 5, where NL is non-linear link, $N_{\text {output }}$ is angle controller code output value. NL is described by the formula:

$$
N_{\mathrm{NL}}=K_{\mathrm{NL}} \cdot \operatorname{sign}\left(N_{\omega}^{\text {calc }}\right),
$$

where $K_{\mathrm{NL}}$ is the value determined at AED adjustment.

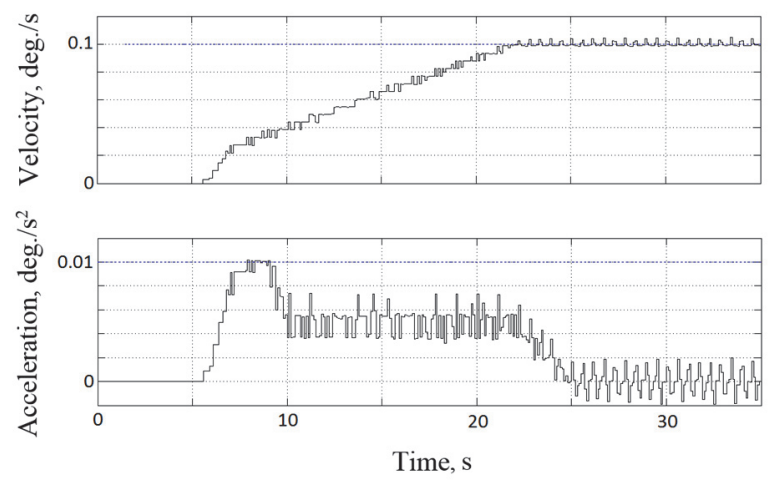

Fig. 4. Output of model of AED operation at the assigned velocity of $0.1 \%$ s set by the generator.

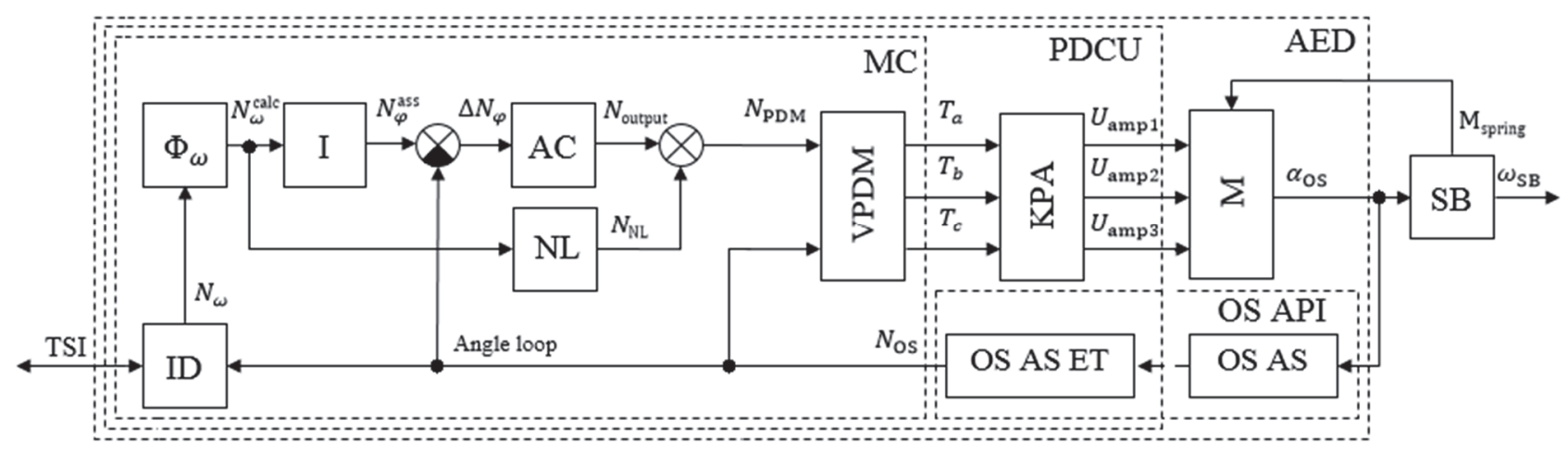

Fig. 5. Block diagram of AED with velocity code generator and non-linear link operating intracker mode based on the varying angle. 
Such value shall not exceed dry friction torque value, but shall compensate its greater part.

Outputs of mathematical model of AED operation with NL and generator of assigned velocity of $0.1 \%$ are shown in Figure 6. It can be seen that the OS pickup time has significantly reduced, and readjustment based on acceleration has decreased.

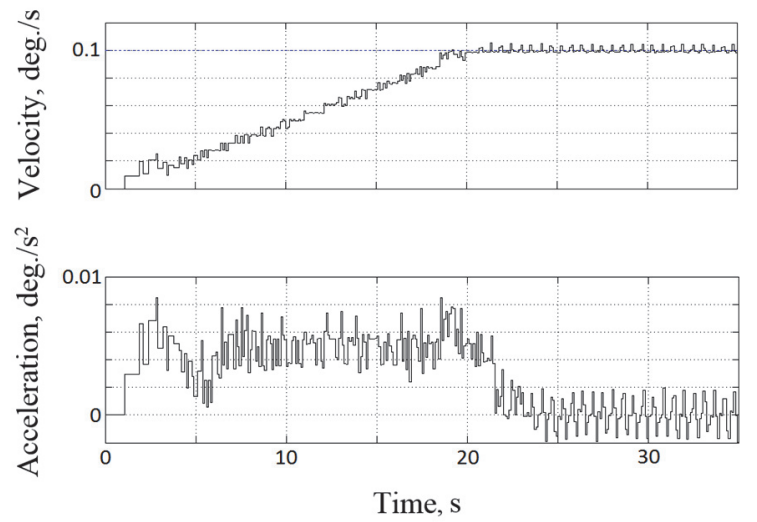

Fig. 6. Outputs of model of AED operation with NL and generator of assigned velocity of $0.1 \%$.

\section{Validation}

Figures 7-8 show the results of experimental operation of SBPD AED with inertial load of the assigned rotation velocities of -0.1 and $-0.0039 \%$, respectively. It can be seen that both acceleration and velocity are controlled by the control system with relatively high accuracy. Inaccuracy of OS angular rotation velocity in the steady state was $1.3 \%$, and $2.56 \%$ for Figures $7-8$, respectively.

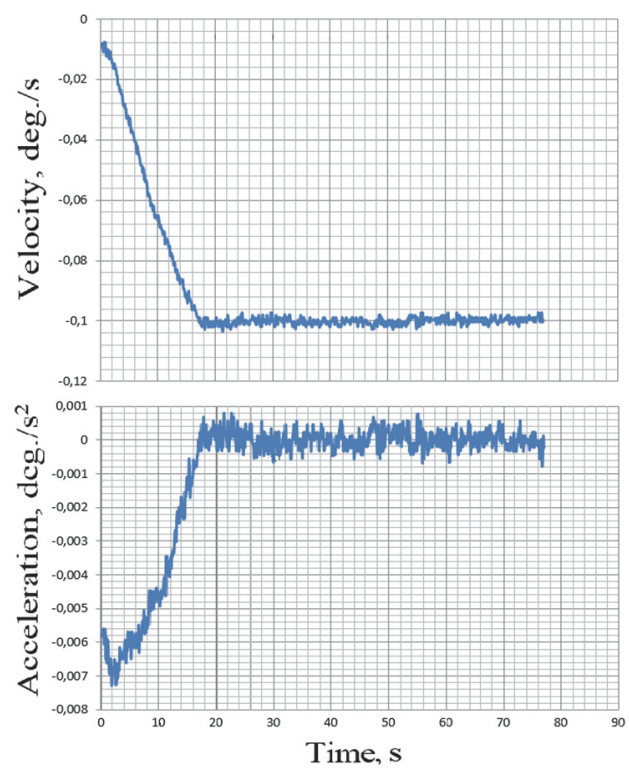

Fig. 7. Real SBPD AED operation at the assigned rotation velocity of $-0.1 \%$.

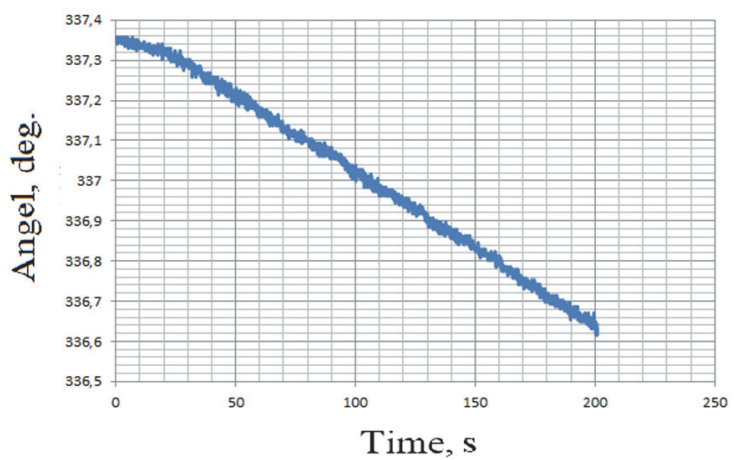

Fig. 8. Real SBPD AED operation at the assigned rotation velocity of $-0.0039 \%$.

\section{Conclusion}

Thus, the results of the SBPD AED modelling and prototype tests demonstrated the effectiveness of the adopted technical solutions. The proposed design of actuating drive ensures accurate control of rotation velocity with controlled acceleration value in the conditions of a large unstable dry friction torque.

\section{References}

1. C. Allegranza, L. Gaillard, R. Le Letty, S. Patti, L. Scolamiero, M. Toso, ACTUATOR 2014, 14th International Conference on New Actuators, 283288 (2014)

2. X. Li, W. Cheng, W. Han, Int. J. Hybrid IT, 7(2) 123-138 (2014)

3. Z.Y. Shu, J.L. Liu, X.X. Zhang, Small and Spec. El. Mach., 41 (2013)

4. A. Kumar, M. Valarmathi, Int. J. Adv. Res. in Electrical, Electronics and Inst. Eng., 2(4), 11971202 (2013)

5. Z. Marinković, D. Marinković, G. Petrović, P. Milić, Teh. V., 19(4), 717-725 (2012)

6. A.S. Arora, G. Singh, IRES 6th Int. Conf., 107-115 (2015)

7. B. You, M. Qiao, J. Xu, D. Liu, Math. Pr. Eng. 2012 (2012)

8. I. Todić, M. Miloš, M. Pavišić, Teh. V., 20(5), 853860 (2013)

9. R.M. Trakhtenberg. Pulse astatic electric drive systems with discrete control (1982)

10. Ch. Phillips, R. Harbor, Feedback control systems (2001) 